\title{
Intensity oscillations observed with Hinode near the south pole of the Sun: leakage of low frequency magneto-acoustic waves into the solar corona
}

\author{
A. K. Srivastava ${ }^{1}$, D. Kuridze ${ }^{2}$, T. V. Zaqarashvilii ${ }^{2}$, and B. N. Dwivedi ${ }^{3}$ \\ 1 Armagh Observatory, College Hill, Armagh BT61 9DG, N. Ireland \\ e-mail: aks@arm.ac.uk \\ 2 Abastumani Astrophysical Observatory at I. Chavchavadze State University, Al Kazbegi ave. 2a, 0160 Tbilisi, Georgia \\ e-mail: [dato; temury]@genao.org \\ 3 Department of Applied Physics, Institute of Technology, Banaras Hindu University, Varanasi-221005, India \\ e-mail: bholadwivedi@gmail.com
}

Received 25 December 2007 / Accepted 21 February 2008

\begin{abstract}
Aims. We study intensity oscillations in the solar chromosphere and corona, above a quiet-Sun magnetic network. Methods. We analyse the time series of He II $256.32 \AA$, Fe XI 188.23 $\AA$ and Fe XII $195.12 \AA$ spectral lines, observed close to the south pole, by the EUV Imaging Spectrometer (EIS), onboard Hinode. We use a standard wavelet tool, to produce power spectra of intensity oscillations above the magnetic network.

Results. For all spectral lines, we detect intensity oscillations of period of approximately seven minutes; and for the He II $256.32 \AA$ line only, we detect an intensity oscillation of period of thirteen minutes, with a probability of approximately $96-98 \%$, which provides the most likely signature of magneto-acoustic wave propagation above the network.

Conclusions. We propose that field-free cavity areas under bipolar magnetic canopies, in the vicinity of a magnetic network, are likely to serve as resonators for the magneto-acoustic waves. The cavities with photospheric sound-speed, and granular dimensions, can produce waves with observed periods. These waves may propagate upwards in the transition region/corona and cause observed intensity oscillations.
\end{abstract}

Key words. Sun: corona - magnetohydrodynamics (MHD) - waves

\section{Introduction}

Complex magnetic fields have an important effect on the dynamics of the solar atmosphere. They channel mechanical energy from the photosphere upwards, and lead to processes such as reflection, channeling or conversion, depending on the field structure and strength. Vertical magnetic tubes support the propagation of tubular (e.g., kink, sausage, torsional) waves, while inclined magnetic fields can cause wave reflection/conversion (Rosenthal et al. 2002) and/or channeling (De Pontieu et al. 2004). Observations support the influence of magnetic fields on the dynamics of waves. For instance, it has been shown that the quiet-Sun magnetic network elements are surrounded by "magnetic shadows", which lack oscillatory power at higher frequencies (McIntosh \& Judge 2001; Krijger et al. 2001; Vecchio et al. 2007). The magnetic shadows probably correspond to socalled "acoustic halos", which are places of increased highfrequency $(>3.3 \mathrm{mHz}$ ) power in the photosphere (Muglach et al. 2005). Thus the high-frequency acoustic waves are reflected (or partially conversed) in the chromosphere. On the other hand, lower-frequency $(<3.3 \mathrm{mHz})$ oscillations are observed intensively over the magnetic network (McAteer et al. 2002; Vecchio et al. 2007), which indicate their penetration into the upper regions. The magnetic-field topology is the most probable explanation for the "wave filtering". The magnetic field is probably vertical inside the network cores, but it has a small-scale closed structure in its vicinity (Schrijver \& Title 2003). Recent observations show small-scale bipolar magnetic fields, above granular cells (de Wijn et al. 2005; Centeno et al. 2007). Kuridze et al. (2007) studied the influence of this small-scale magnetic canopy, on the wave dynamics. They found that the field-free cavity regions, under the canopy, may trap high-frequency acoustic oscillations, while the lower-frequency oscillations may be channeled upwards in the form of magneto-acoustic waves.

In this paper, we study the spectrum of intensity oscillations above the quiet-Sun magnetic network, using new data acquired using EIS onboard Hinode. The observational results are interpreted using magnetohydrodynamic (MHD) modeling of wave dynamics, in a cylindrical bipolar magnetic canopy.

\section{Observations and data reduction}

We use time series data of the on-disk quiet-Sun, close to its south pole, as observed by the $40^{\prime \prime}$-slot of EIS (Culhane et al. 2006). The $40^{\prime \prime}$ and $266^{\prime \prime}$ slots are appropriate for image analyses using light curves, while $1^{\prime \prime}$ and $2^{\prime \prime}$ slits are ideal for spectral and Doppler analyses using spectral line profiles. EIS observes high resolution spectra in two wavelength intervals 170-211 $\AA$ and 246-292 $\AA$, by short-wavelength (SW) and long-wavelength (LW) CCDs respectively. The spectral resolution of EIS is $0.0223 \AA$ per pixel. There are approximately 


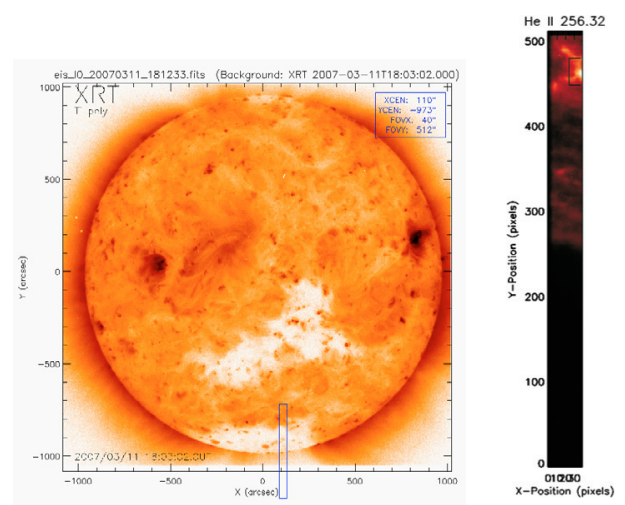

Fig. 1. The pointing position of $40^{\prime \prime}$ slot over the full disk image of XRT/Hinode (left), and the brightened magnetic network is shown in He II $256.32 \AA$ line (right). The position on the full disc image is expressed in arc sec and the position on the slot in pixels.

16 pixels and 2 pixels offsets, in the $Y$ and $X$-coordinates respectively of two CCDs (Young et al. 2007). Observations were acquired on 11th March, 2007, and the name of our study is HPW005_QS_Slot_60m. The data contain He II $256.32 \AA$,

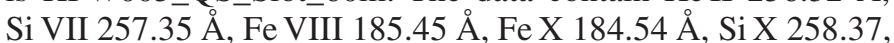
$261.04 \AA$, Fe XI 188.23 $202.04 \AA$, Fe XIV 264.78, 274.2 A, Fe XV 284.12 Å, Fe XVI $262.92 \AA$, and Ca XVII 192.82 A spectral lines. The observations started at 18:12:33 UT, and ended at 19:04:06 UT. The position of the $X$-centre and $Y$-centre of the slot, were $110^{\prime \prime}$ and $-973^{\prime \prime}$ respectively, while the $X$-FOV and $Y$-FOV, were $40^{\prime \prime}$ and $512^{\prime \prime}$ respectively (Fig. 1). The binning of the data was $1^{\prime \prime} \times 1^{\prime \prime}$. The exposure time for each spectral line was $60 \mathrm{~s}$, and the integration time for each step of the time series was uniform, being $60 \mathrm{~s}$. We choose the brightest core of the quiet-Sun magnetic network, in three spectral lines: He II $256.32 \AA$, Fe XI $188.86 \AA$, and Fe XII $195.12 \AA$. He II was observed using the LW CCD $\left[\left(X_{\mathrm{LW}}, Y_{\mathrm{LW}}\right)=\right.$ (36th pixel, 461th pixel) $)$, while the iron lines were observed using the SW CCD. We can therefore compensate, approximately, for the offset of the iron lines, using the relation: $X_{\mathrm{SW}}=\left(X_{\mathrm{LW}}-2\right), Y_{\mathrm{SW}}=\left(Y_{\mathrm{LW}}+16\right)$.

We apply standard EIS data-reduction procedures, to the data acquired at the telescope (the raw (zeroth level) data).The subroutines can be found in the SSWIDL software tree ${ }^{1}$. These standard subroutines correct for dark-current subtraction, cosmic-ray removal, flat-field correction, hot pixels, and bad/missing pixels. The data is saved in the level-1 data file, while associated errors are saved in the error file. The signal-to-noise ratios $(\mathrm{S} / \mathrm{N})$ of selected lines (He II 256.32 A, Fe XI 188.23 A, Fe XII 195.12 ̊) is in the range $20-50$, which is adequate for our analyses. The summation of $X$ or $Y$ pixels was not completed, to prevent information losses about small bright features in the magnetic network.

\section{Results}

We use the Morlet wavelet tool, to produce the power spectrum of oscillations, in different spectral lines. We note that the Morlet wavelet suffers from the edge effect that is typical of time series data. This effect, however, is significant in regions defined to be a cone of influence (COI). The wavelet procedure, its noise filtering, and COI effects are described in Torrence \& Compo (1998). A randomisation technique evaluates the peak power in

1 e.g., www.darts.isas.jaxa.jp/pub/solar/ssw/hinode/eis/

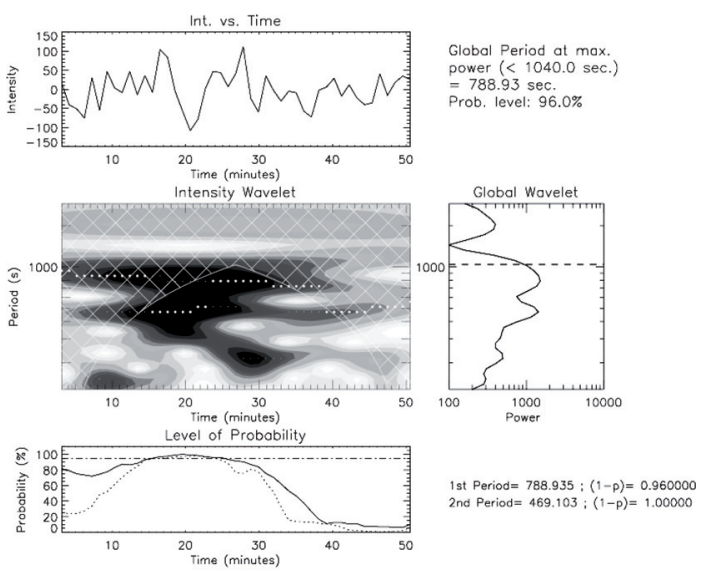

Fig. 2. The wavelet result for He II $256.32 \AA$ line: the top panel shows the variation of intensity, the wavelet power spectrum is given in the middle panel, and the probability is given in the bottom panel.

the global wavelet spectrum, which is the average peak power over time, and is equivalent to a smoothed Fourier power spectrum. This technique compares the average values to peak powers evaluated for $n$ ! equally-likely permutations of the time series data, assuming that $n$ values of measured intensities are independent of $n$ measured times, if no periodic signal is present.The proportion of permutations that provides a value greater or equal to the original peak power of the time series, will provide the probability that there is no periodic component $(p)$. The percentage probability that periodic components are present in the data, will be $(1-p) \times 100$, and for real oscillations, the lowest acceptable probability is $95 \%$. We calculated 200 permutations of the reliable estimation of $p$, and hence the probability of real oscillations. The details of the randomisation technique used to obtain statistically-significant real oscillation periods, were given by Nemec \& Nemec (1985) and O'Shea et al. (2001). We do not remove any upper/longer period intervals or associated powers of our time series data, during the wavelet analysis. We do not consider, however, the first 3-4 min of the data analysed, because this was associated with an abrupt intensity enhancement. We choose the "running average" option of O'Shea's wavelet tool, and smooth the original signal by a window of scalarwidth " 10 ". We use an average smoothing technique, based on low-pass filtering methods, to reduce noise in the original signal before measuring the real periodicity. The maximum allowed period from COI, where the edge effect is more effective, is $1039 \mathrm{~s}$. Hence, the power reduces substantially beyond this threshold. In our wavelet analysis, we consider only the power peaks and corresponding real periods below this threshold. The results of the wavelet analysis, for the spectral lines He II $256.32 \AA$, Fe XI 188.23 $\AA$ and Fe XII 195.12 $\AA$, are provided in Figs. 3-5. We measured periodicity of $\sim 6.5-7.5 \mathrm{~min}$, with a probability of $96-98 \%$, in all spectral lines, and a periodicity of $\sim 13 \mathrm{~min}$ of the He II line. It should be noted that both the $\sim 7.5 \mathrm{~min}$ and $\sim 13$ min periodicities are statistically-significant in the chromospheric He II line. These oscillations are a signature of the propagation of low-frequency magneto-acoustic waves, close to the magnetic network. We performed the wavelet analysis in the non-network and non-brightened region. We chose the coordinate $\left(X_{\mathrm{SW}}, Y_{\mathrm{SW}}\right) \approx(26,326)$ for iron lines, and corresponding offset-compensated coordinates for the He II line. The wavelet method measures a periodicity of $\approx 13-16 \mathrm{~min}$, with a probability between $77 \%$ and $83 \%$, which is not a real oscillation according to O'Shea et al. (2001). Hence, $\sim 7 \mathrm{~min}$ and $\sim 13 \mathrm{~min}$ 


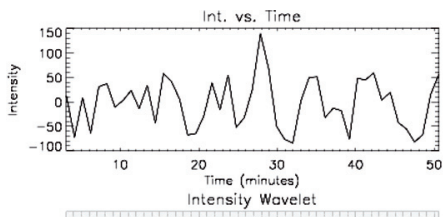

Globol Period ot mox.
power (< 1040.0 sec.) $=394.47 \mathrm{sec}$.
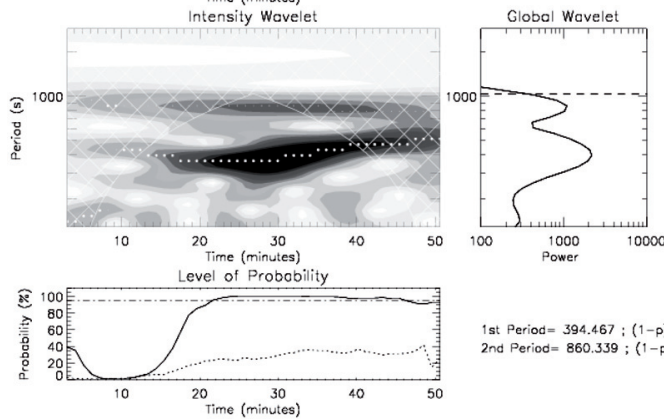

1st Period $=394.467:(1-p)=0.980000$
2nd Period $=860.339 ;(1-p)=1.00000$

Fig. 3. The wavelet result for Fe XI $188.23 \AA$ line: the top panel shows the variation of intensity, the wavelet power spectrum is given in the middle panel, and the probability is given in the bottom panel.
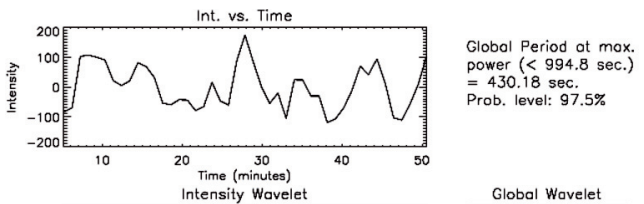

$=430.18 \mathrm{sec}$.
$=4$.
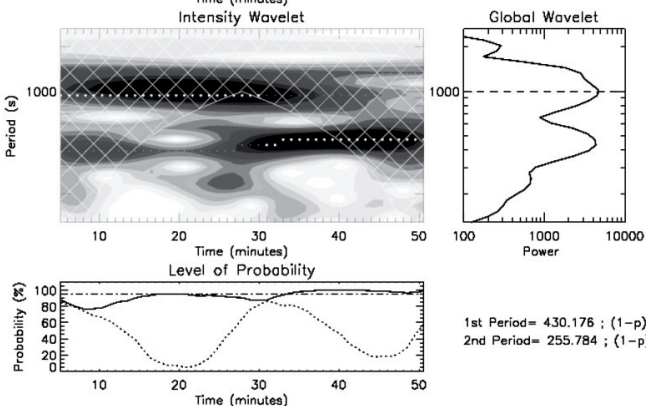

1st Period $=430.176:(1-p)=0.975000$
2nd Period= $255.784 ;(1-p)=1.00000$

Fig. 4. The wavelet result for Fe XII $195.12 \AA$ line: the top panel shows the variation of intensity, the wavelet power spectrum is given in the middle panel, and the probability is given in the bottom panel.

oscillations are only apparent in the brightened magnetic core region.

\section{A theoretical model}

As already noted, observed intensity oscillations in different spectral lines over the magnetic network, carry the signature of the propagation of magneto-acoustic waves from the photosphere. The observed frequencies are, however, below the photospheric cut-off value. The waves should not propagate for very long, upwards through the lower atmosphere. Their observation in the upper atmosphere is therefore difficult to explain. An explanation can be found if the waves are generated in situ, or if they propagate at an arbitrary angle, which would reduce the cutoff frequency (De Pontieu et al. 2004). Here, we present a theoretical model, which supports the interpretation that the waves propagate upwards from the photosphere.

The magnetic field is probably vertical in the core of network, but becomes inclined in the vicinity of small-scale bipolar loops (McIntosh \& Judge 2001; Schrijver \& Title 2003). Fieldfree cavities of granular dimensions under this small-scale cylindrical canopy may become effective resonators for MHD waves. Higher-order harmonics may be trapped in the cavity, but the first harmonic may propagate upwards. For all harmonics, the

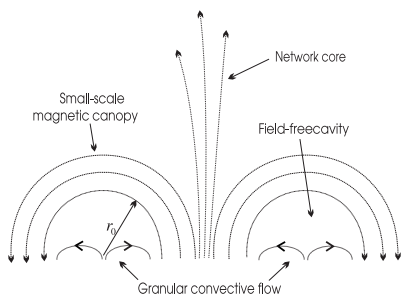

Fig. 5. Schematic view of a small-scale bipolar magnetic canopy overlying a field-free cavity region near a network core.

photospheric cut-off frequency is lower than the actual cut-off value, due to a finite wave-number component in the horizontal direction.

A cylindrical bipolar magnetic field model, similar to that developed by McIntosh \& Judge (2001) and Schrijver \& Title (2003), is used to reproduce the wave behaviour in the vicinity of the magnetic network. We suggest that a field-free cavity area ( $r<r_{0}$ region in Fig. 5), overlayed by the magnetic canopy, can be formed over granular cells, when plasma flows transport the magnetic flux, at boundaries (Centeno et al. 2007). The magnetic field, $B_{0}$, in the overlying canopy ( $r>r_{0}$ region in Fig. 5) is proposed to be current-free, with only a $\phi$-component, in a cylindrical coordinate system $(r, \phi, z)$, i.e. $\boldsymbol{B}_{0}=\left(0, B_{\phi}(r), 0\right)$, where $B_{\phi}(r)=B_{\phi 0} r_{0} / r$ (Díaz et al. 2006).

The oscillation spectrum can be obtained by solving governing plasma equations separately in both, the canopy and a cavity regions. The merging of the solutions at the canopy/cavity interface, then provides the dispersion relation for oscillations.

A Fourier analysis of the linearized adiabatic hydrodynamic equations, with respect to both the time $(t)$ and coordinate $(\phi)$, leads to the Bessel equation for a field-free cavity region:

$\frac{\partial^{2} \rho_{1}}{\partial r^{2}}+\frac{1}{r} \frac{\partial \rho_{1}}{\partial r}+\left[\frac{\omega^{2}}{c_{0}^{2}}-\frac{m^{2}}{r^{2}}\right] \rho_{1}=0$,

where $m$ corresponds to the azimuthal wave number, $\rho_{1}$ is the density perturbations, and $c_{0}=\sqrt{\gamma p_{0} / \rho_{01}}$ is the adiabatic sound speed. Here, $p_{0}$ and $\rho_{01}$ are unperturbed pressure and density in the cavity $\left(r<r_{0}\right)$ and $\gamma$ is the ratio of specific heats. The solution of this equation, is the Bessel function $J_{m}\left(k_{1} r\right)$, where $k_{1}=\omega / c_{0}$.

In the magnetic canopy region $\left(r>r_{0}\right)$, we use the coldplasma approximation. In this case, linearised MHD equations lead to:

$\frac{\partial^{2} \xi_{r}}{\partial r^{2}}-\frac{1}{r} \frac{\partial \xi_{r}}{\partial r}+\left[\frac{\omega^{2}}{v_{\mathrm{A}}^{2}(r)}+\frac{1}{r^{2}}-\frac{m^{2}}{r^{2}}\right] \xi_{r}=0$,

where $\xi_{r}$ is the transverse Lagrangian position-vector component, and $v_{\mathrm{A}}=B_{\phi} / \sqrt{4 \pi \rho_{02}}$, with $\rho_{02}$ being the unperturbed density inside the canopy region. The solution of this equation is proportional to the Hankel function of half-integer order $r H_{m / 2}\left(k_{2} r\right)$, where $k_{2}=\omega / 2 v_{\text {A }}$ (Díaz et al. 2006).

The continuity of the transverse Lagrangian position-vector component, and the total Lagrangian pressure perturbation at the canopy/cavity interface $r=r_{0}$, leads to the general dispersion relation (Kuridze et al. 2007):

$\frac{\omega^{2}}{v_{\mathrm{A}}^{2}} \frac{\rho_{01}}{\rho_{02}} \frac{J_{m}\left(k_{1} r_{0}\right)}{k_{1} J_{m}^{\prime}\left(k_{1} r_{0}\right)}=-\frac{k_{2} r_{0} H_{m / 2}^{\prime}\left(k_{2} r_{0}\right)}{r_{0} H_{m / 2}\left(k_{2} r_{0}\right)}$.

Equation (3) describes the oscillation spectrum in the cavity region under the magnetic canopy (Fig. 5). The wave frequency 


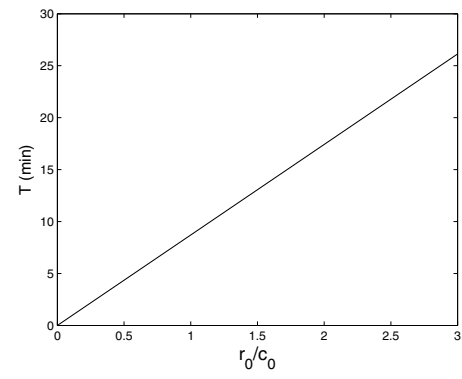

Fig. 6. The period of $m=1$ harmonic vs. the ratio of cavity size and sound speed $r_{0} / c_{0} . r_{0} / c_{0}$ is normalised by $1 \mathrm{~min}$. The observed wave periods $\sim 7$ and $13 \mathrm{~min}$ correspond to the radius of $\sim 450$ and $800 \mathrm{~km}$ for the sound speed of $9 \mathrm{~km} \mathrm{~s}^{-1}$.

is, in general, complex, which causes the leakage upwards of oscillations. Kuridze et al. (2007) showed that higher-order $m>1$ harmonics are trapped in the cavity, while the first $m=1$ harmonic is leaky. This implies that the first harmonic can propagate upwards, as a fast magneto-acoustic wave, and create intensity oscillations in coronal spectral lines. In this context, we emphasize the behaviour of the $m=1$ harmonic.

Figure 6 shows the dependence of the period of $m=1$ harmonic, on the ratio of cavity radius $r_{0}$, and sound speed $c_{0}$. Hence, the typical photospheric sound-speed, and the cavity radius determine the oscillation period. We infer from Fig. 6 that the photospheric sound-speed, $c_{0}=9 \mathrm{~km} \mathrm{~s}^{-1}$, implies an observed period of $\sim 6.5-7.5 \mathrm{~min}$ for the cavity diameter of $\sim 800-900 \mathrm{~km}$, and $\sim 13 \mathrm{~min}$ for the diameter of $\sim 1600 \mathrm{~km}$. This probably leads to the enhanced power at granular radii, on the same scale. Photometric and spectroscopic study of abnormal granulations close to strong magnetic fields, does indeed display enhanced power, at granular diameters of $1.3^{\prime \prime}$ $(\sim 900 \mathrm{~km})$, and $2.5^{\prime \prime}(\sim 1800 \mathrm{~km})$ (Sobotka et al. 1994). The enhanced power, on the scale of granular size, and close to the magnetic network core, can explain the oscillation periods measured.

\section{Discussion and conclusions}

The analysis of new observational data, obtained by EIS, onboard Hinode, show $\sim 7$ min intensity oscillations in He II 256.32 $\AA$, Fe XI 188.23 $\AA$ and Fe XII 195.12 $\AA$ spectral lines, with a probability of $96-98 \%$, above the magnetic network, close to the south pole. We also found $\sim 13$ min intensity oscillations in He II $256.32 \AA$. The oscillations were observed independently, in three spectral lines, corresponding to different plasma temperatures: $\log T_{\mathrm{He} I \mathrm{I}}=4.90, \log T_{\mathrm{FeXI}}=6.10$, and $\log T_{\mathrm{Fe} \text { XII }}=6.20$. The oscillations are probably present at different heights, which implies that the wave propagates from the photosphere, into the transition region and the corona.

We suggest for the first time that the field-free cavity regions, under the bipolar small-scale magnetic canopy, in the vicinity of the network core, may serve as resonators for the waves. This magnetic field configuration has been suggested to be the case, close to the network core (McIntosh \& Judge 2001; Schrijver \& Title 2003), and above granular cells (de Wijn et al. 2005; Centeno et al. 2007). The field-free cavities, under the bipolar magnetic canopy, probably have granular dimensions, as the granular flow transport the magnetic flux, at the cell boundaries. We solve the MHD equations in the cavity and canopy regions, separately, and merge the solutions at the cavity-canopy interface. Consequently, we obtain the dispersion relation for oscillations, which shows that the first harmonic is leaky, and that the oscillations may propagate upwards. The period of the first harmonic depends on both the cavity radius, and the sound speed. Measuring periods of $\sim 7 \mathrm{~min}$ and $\sim 13 \mathrm{~min}$, implies that the cavity diameter should be $\sim 900$ and $\sim 1600 \mathrm{~km}$ respectively, for a photospheric sound speed of $\sim 9 \mathrm{~km} \mathrm{~s}^{-1}$. This result determines the power spectra of the abnormal granulation, which shows enhanced power at granular diameters of $900 \mathrm{~km}$ and $1800 \mathrm{~km}$ (Sobotka et al. 1994). The observed oscillations can be explained by wave excitation in cavities under the small-scale canopy. It should be mentioned, that the frequency of the first harmonic is above the photospheric cut-off frequency, because of the horizontal component of wave vector. Therefore, the oscillation may easily penetrate into higher regions. Inclusion of gravitational stratification is, however, necessary to demonstrate a clearer link between observations and theory.

Oscillations of period $\sim 7-13 \mathrm{~min}$ are observed, only in the brightest core of the magnetic network. We do not find statistically-significant oscillations in nearby non-magnetic regions. Therefore, it appears that the oscillations are typical of magnetic networks. Similar analyses should also be completed above other network elements. Such future investigations will analyse Hinode and ground-based observational data.

Acknowledgements. A.K.S. thanks Prof. J.G. Doyle for valuable discussions and encouragements, and Dr. E. O'Shea for "Randomlet". T.V.Z. and D.K. thank Prof. S. Poedts and Dr. B. Shergelashvili for valuable discussions. This work was supported by the grant of Georgian National Science Foundation GNSF/ST06/4098. Hinode is a Japanese mission developed and launched by ISAS/JAXA, with NAOJ as domestic partner and NASA and STFC (UK) as international partners. It is operated by these agencies in co-operation with ESA and NSC (Norway). We also acknowledge Dr. Harry Warren for his observing sequence, which is avialable in EIS data archive. We thank the referee for his valuable suggestions, which considerably improved the manuscript.

\section{References}

Centeno, R., Socas-Navarro, H., Lites, B., et al. 2007, ApJ, 666, L137 Culhane, J. L., Doschek, G. A., Watanabe, T., et al. 2006, SPIE, 6266, 62660 De Pontieu, B., Erdélyi, R., \& James, S. P. 2004, Nature, 430, 536 Díaz, A. J., Zaqarashvili, T., \& Roberts, B. 2006, A\&A, 455, 709 Krijger, J. M., Rutten, R. J., Lites, B. W., et al. 2001, A\&A, 379, 1052 Kuridze, D., Zaqarashvili, T. V., Shergelashvili, B. M., \& Poedts, S. 2007, Annales Geophysicae, accepted

Lawrence, J. K., Cadavid, A. C., Miccolis, D., Berger, T. E., \& Ruzmaikin, A. 2003, ApJ, 597, 1178

Linnell Nemec, A. F., \& Nemec, J. M. 1985, AJ, 90, 2317

McAteer, R. T. J., Gallagher, P. T., Williams, D. R., et al. 2002, ApJ, 567, L165 McIntosh, S. W., \& Judge, P. G. 2001, ApJ, 561, 420

Muglach, K., Hofmann, A., \& Staude, J. 2005, A\&A, 437, 1055

O'Shea, E., Banerjee, D., Doyle, J. G., Fleck, B., \& Murtagh, F. 2001, A\&A, 368,1095

Rosenthal, C. S., Bogdan, T. J., Carlsson, M., et al. 2002, ApJ, 564, 508

Schrijver, C. J., \& Title, A. M. 2003, ApJ, 597, L165

Sobotka, M., Bonet, J. A., \& Vazquez, M. 1994, ApJ, 426, 404

Torrence, C., \& Compo, G. P. 1998, BAMS, 79, 61

Vecchio, A., Cauzzi, G., Reardon, K. P., Janssen, K., \& Rimmele, T. 2007, A\&A, 461, L1

de Wijn, A. G., Rutten, R. J., Haverkamp, E. M. W. P., \& Sutterlin, P. 2005, A\&A, 441, 1183

Young, P. R., Del Zanna, G., Mason, H. E., et al. 2007, PASJ, 59, 857 